\title{
On the $\mathcal{S}_{n}$-modules generated by partitions of a given shape
}

\author{
Daniel Kane ${ }^{1}$ and Steven Sivek ${ }^{2}$ \\ ${ }^{1}$ Department of Mathematics, Harvard University \\ dankane@math.harvard.edu \\ ${ }^{2}$ Department of Mathematics, Massachusetts Institute of Technology \\ ssivek@math . mit . edu
}

Submitted: May 12, 2008; Accepted: Aug 24, 2008; Published: Aug 31, 2008

Mathematics Subject Classification: 05E10

\begin{abstract}
Given a Young diagram $\lambda$ and the set $H^{\lambda}$ of partitions of $\{1,2, \ldots,|\lambda|\}$ of shape $\lambda$, we analyze a particular $\mathcal{S}_{|\lambda|}$-module homomorphism $\mathbb{Q} H^{\lambda} \rightarrow \mathbb{Q} H^{\lambda^{\prime}}$ to show that $\mathbb{Q} H^{\lambda}$ is a submodule of $\mathbb{Q} H^{\lambda^{\prime}}$ whenever $\lambda$ is a hook $(n, 1,1, \ldots, 1)$ with $m$ rows, $n \geq m$, or any diagram with two rows.
\end{abstract}

\section{Introduction}

Let $\lambda=\left(\lambda_{1}, \ldots, \lambda_{n}\right)$ be a Young diagram and let $H^{\lambda}$ be the set of row tabloids of shape $\lambda$ : that is, $H^{\lambda}$ is the set of all ways to fill the diagram with the numbers $1,2, \ldots,|\lambda|=\sum \lambda_{i}$ such that the order within a given row does not matter and neither does the order of two rows of the same size. Let $V^{\lambda}$ denote the set of column tabloids of shape $\lambda$, which are defined analogously, and note that $V^{\lambda} \cong H^{\lambda^{\prime}}$ canonically. In particular, $H^{\lambda}$ and $V^{\lambda}$ are the sets of partitions of $\{1,2, \ldots,|\lambda|\}$ of shape $\lambda$ and $\lambda^{\prime}$, respectively. Each of these sets has an obvious $\mathcal{S}_{|\lambda|}$ action obtained by permuting the numbers which fill the tabloids. For any two tabloids $\Gamma \in H^{\lambda}$ and $\Delta \in V^{\lambda}$ we define an orthogonality relation $\Gamma \perp \Delta$ to be satisfied precisely when $|r \cap c| \leq 1$ for every row $r$ of $\Gamma$ and column $c$ of $\Delta$.

We define a homomorphism of $\mathcal{S}_{|\lambda|}$-modules $\mathbb{Q} V^{\lambda} \rightarrow \mathbb{Q} H^{\lambda}$ in terms of a matrix $K^{\lambda}$, whose rows and columns are indexed by $H^{\lambda}$ and $V^{\lambda}$ respectively and whose entries $K_{\Gamma, \Delta}^{\lambda}$ are equal to 1 whenever $\Gamma \perp \Delta$ and 0 otherwise. Then Pylyavskyy [5] conjectured that $K^{\lambda}$ has full rank, modifying a false conjecture of Stanley [7]. This is of particular interest due to work of Black and List [1], in which they introduced this homomorphism in the case where $\lambda$ is an $n \times m$ rectangle and showed that the conjecture would imply Foulkes' plethysm conjecture as follows: 
Proposition 1.1 (Black and List). If $K^{n \times m}$ has full rank for some $n \geq m>1$, then the $\mathcal{S}(r n)$-module $\mathbb{Q} H^{n \times r}$ contains $\mathbb{Q} H^{r \times n}$ as a submodule for $1 \leq r \leq m$.

Unfortunately, this will not suffice to prove the full conjecture, since Mller and Neunhffer have shown that $K^{5 \times 5}$ does not have full rank [4]. Furthermore, the second author showed Pylyavskyy's conjecture to be false in infinitely many cases: any diagram $\lambda$ can be nested in some larger $\mu$ such that $K^{\mu}$ does not have full rank, and there are symmetric shapes $\lambda_{n}$ such that $\frac{\operatorname{rank}\left(K^{\lambda}\right)}{\left|H^{\lambda}\right|} \rightarrow 0$ as $n \rightarrow \infty[6]$.

This paper is concerned with proving positive results about the matrices $K^{\lambda}$. In order to show that certain $K^{\lambda}$ have full rank, we construct a related matrix:

Definition 1.2. Let $M^{\lambda}=\left(K^{\lambda}\right)\left(K^{\lambda}\right)^{\top}$ for all shapes $\lambda$ such that $\left|H^{\lambda}\right| \leq\left|V^{\lambda}\right|$.

This matrix was diagonalized by Coker [2] in the rectangular cases $\lambda=(n, n)$ as well as $\lambda=(m, m, m)$ for $3 \leq m \leq 6$. Note that $M^{\lambda}$ is square (specifically $\left|H^{\lambda}\right| \times$ $\left.\left|H^{\lambda}\right|\right)$ and symmetric, hence diagonalizable, and it is positive semidefinite since $v^{\top} M^{\lambda} v=$ $\left|\left(K^{\lambda}\right)^{\top} v\right|^{2} \geq 0$ for any vector $v \in \mathbb{Q} H^{\lambda}$. Furthermore, it is easy to see from the definition that the entries of $M^{\lambda}$ are indexed by pairs of row tabloids $\Gamma_{1}, \Gamma_{2} \in H^{\lambda}$, and that $M_{\Gamma_{1}, \Gamma_{2}}^{\lambda}=$ $\#\left\{\Delta \in V^{\lambda} \mid \Gamma_{1}, \Gamma_{2} \perp \Delta\right\}$.

Proposition 1.3. Suppose that $\left|H^{\lambda}\right| \leq\left|V^{\lambda}\right|$. Then the matrix $M^{\lambda}$ is invertible if and only if $\left(K^{\lambda}\right)^{\top}: \mathbb{Q} H^{\lambda} \rightarrow \mathbb{Q} V^{\lambda}$ is injective.

Proof. If $\left(K^{\lambda}\right)^{\top}$ is injective, then for any nonzero vector $v \in \mathbb{Q} H^{\lambda}$ we have $v^{\top} M^{\lambda} v=$ $\left|\left(K^{\lambda}\right)^{\top} v\right|^{2}>0$, hence $M^{\lambda} v \neq 0$; this implies that $M^{\lambda}$ is invertible. If however $\left(K^{\lambda}\right)^{\top} v=0$ for some nonzero $v$, then $M^{\lambda} v=K^{\lambda}\left(\left(K^{\lambda}\right)^{\top} v\right)=0$ and so $M^{\lambda}$ is not invertible.

With this proposition in mind, we will show in the following sections that $M^{\lambda}$ is invertible for several classes of Young diagrams, namely hooks and diagrams with two rows, using a construction which is nearly identical in both cases. The case of hooks is already known but seems to be unpublished (see [7, Problem 9]), and our proof in this case is both new and relatively simple. It is therefore good preparation for the main result, which establishes for the first time that $\mathbb{Q} H^{\lambda}$ is a submodule of $\mathbb{Q} H^{\lambda^{\prime}}$ whenever $\lambda$ is a diagram with two rows.

\section{Hooks}

An $n \times m$ hook is defined as the Young diagram $\lambda=(n, 1, \ldots, 1)$ with a total of $m$ rows; we will write it as $\langle n, m\rangle$ for convenience. Since any row tabloid $\Gamma \in H^{\langle n, m\rangle}$ is uniquely determined by its top row, we can think of these interchangeably as row tabloids and $n$-element subsets of $\{1,2, \ldots, n+m-1\}$. We will also define a function $\delta\left(\Gamma_{1}, \Gamma_{2}\right)$ as the number of elements in the intersection of these subsets, so for example when $n=5$ and $m=3$ we have $\delta(\{1,2,3,5,6\},\{1,2,4,6,7\})=3$.

In this section we will describe the matrix $M^{\langle n, m\rangle}, n \geq m$, and prove that it is invertible, a result that was claimed without proof in [7]. The diagonal entries $M_{\Gamma, \Gamma}^{\langle n, m\rangle}$ are 
clearly $n$, since each of these is the number of $\Delta \in V^{\langle n, m\rangle}$ which are orthogonal to $\Gamma$; in any such $\Delta$, the $n$ elements in the top row of $\Gamma$ must each be in a different one of the $n$ columns of $\Delta$, and we may choose exactly one of them to be in the $m$-element first column. Otherwise, suppose that $\delta\left(\Gamma_{1}, \Gamma_{2}\right)=k<n$. Any column tabloid $\Delta$ which is orthogonal to both $\Gamma_{1}$ and $\Gamma_{2}$ must place one element from the top row of each $\Gamma_{i}$ in each column, so each of the $n-1$ elements in the 1-element columns of $\Delta$ must be in both the top rows of $\Gamma_{1}$ and $\Gamma_{2}$ : that is, if $\delta\left(\Gamma_{1}, \Gamma_{2}\right)<n-1$ then there is no $\Delta \in V^{\langle n, m\rangle}$ which is orthogonal to both. Otherwise, if $\delta\left(\Gamma_{1}, \Gamma_{2}\right)=n-1$ then the $n-1$ elements common to their top rows must fill all of the 1-element columns of $\Delta$, and this uniquely determines the remaining $m$-element column of $\Delta$, so there is exactly one $\Delta$ orthogonal to both of the $\Gamma_{i}$. In summary, we have

$$
M_{\Gamma_{1}, \Gamma_{2}}^{\langle n, m\rangle}=\left\{\begin{array}{cc}
n & \text { if } \Gamma_{1}=\Gamma_{2} \\
1 & \text { if } \delta\left(\Gamma_{1}, \Gamma_{2}\right)=n-1 \\
0 & \text { otherwise. }
\end{array}\right.
$$

We may attempt to show that $M^{\langle n, m\rangle}$ is invertible by diagonalizing it. A series of related computations using a combination of Sage and LinBox [8,3] suggested many of the following results.

Lemma 2.1. The minimal polynomial of $M^{\langle n, m\rangle}$ has degree at most $m$.

Proof. Suppose that $P$ and $Q$ are two matrices whose entries are $P_{\Gamma_{1} \Gamma_{2}}=p_{k}$ and $Q_{\Gamma_{1} \Gamma_{2}}=$ $q_{k}$ whenever $\delta\left(\Gamma_{1}, \Gamma_{2}\right)=k$. Then if $R=P Q$, we have

$$
R_{\Gamma_{1} \Gamma_{2}}=\sum_{\Gamma_{3}} P_{\Gamma_{1} \Gamma_{3}} Q_{\Gamma_{3} \Gamma_{2}}
$$

If indeed $\delta\left(\Gamma_{1}, \Gamma_{2}\right)=k$, then we may apply some permutation $\sigma \in \mathcal{S}_{n+m-1}$ which will take $\Gamma_{1}$ to the tabloid with top row $\{1,2, \ldots, n\}$ and $\Gamma_{2}$ to the tabloid with top row $\{1,2, \ldots, k, n+1, \ldots, 2 n-k\}$, and it is easy to verify that the action of $\sigma$ preserves the value of $\delta$ and hence the terms $P_{\Gamma_{1} \Gamma_{3}}$ and $Q_{\Gamma_{3} \Gamma_{2}}$, so $R_{\Gamma_{1} \Gamma_{2}}$ depends not on the actual tabloids $\Gamma_{i}$ but on $\delta\left(\Gamma_{1}, \Gamma_{2}\right)$. In particular, we may define sequences $a_{k}^{e}, n-m+1 \leq k \leq n$, so that $\left(M^{\langle n, m\rangle}\right)^{e}$ has $\left(\Gamma_{1}, \Gamma_{2}\right)$-entry equal to $a_{e, k}$ whenever $\delta\left(\Gamma_{1}, \Gamma_{2}\right)=k$.

Now consider an arbitrary polynomial $f(x)=\sum_{i=0}^{m} c_{i} x^{i}$. The entries of $f\left(M^{\langle n, m\rangle}\right)$ are all of the form $\sum_{i=0}^{m} c_{i} a_{i, k}$, and there are $m$ possible values of $k$ but $m+1$ coefficients $c_{i}$, so there is some nontrivial choice of the $c_{i}$ which makes each sum $\sum c_{i} a_{i, k}$ equal to zero. Therefore we can find a nonzero polynomial $f$, deg $f \leq m$, such that $f\left(M^{\langle n, m\rangle}\right)=0$.

Since $M^{\langle n, m\rangle}$ is diagonalizable, its minimal polynomial is equal to the product $\prod(x-\lambda)$ over all distinct eigenvalues $\lambda$ of $M^{\langle n, m\rangle}$. This means that the matrix has at most $m$ distinct eigenvalues, and in fact we can compute all of them:

Proposition 2.2. The eigenvalues of $M^{\langle n, m\rangle}$ are precisely the values $(n-k)(m-k)$ for $0 \leq k<m$. 
Proof. We will explicitly construct eigenvectors $v_{k}=\left(a_{k, \Gamma}\right)_{\Gamma \in H^{\langle n, m\rangle}}$ for each value of $k$, $0 \leq k<m$, referring to each tabloid $\Gamma$ by the set of elements in its first row for convenience. Declare an $n$-element subset of $\{1,2, \ldots, n+m-1\}$ to be $k$-ordinary if it contains zero or two elements of any one of the pairs $\{1,2\},\{3,4\}, \ldots,\{2 k-1,2 k\}$. A set which is not $k$-ordinary, i.e. which has exactly one element from each of those pairs and thus $n-k$ elements of $\{2 k+1, \ldots, n+m-1\}$, is called $k$-even if it contains an even number of odd elements up to $2 k$ and $k$-odd otherwise. (Note that all $n$-element subsets are $k$-even when $k=0$.) Then for each $\Gamma$, we set $a_{k, \Gamma}=0$ if $\Gamma$ is $k$-ordinary, $a_{k, \Gamma}=1$ if $\Gamma$ is $k$-even, and $a_{k, \Gamma}=-1$ if $\Gamma$ is $k$-odd. Write $\left(b_{\Gamma}\right)=M^{\langle n, m\rangle}\left(a_{k, \Gamma}\right)$; we wish to show that $b_{\Gamma}=(n-k)(m-k) a_{k, \Gamma}$ for all $\Gamma$.

Suppose that $\Gamma$ is $k$-ordinary. Then $b_{\Gamma}=\sum_{\Gamma^{\prime} k \text {-even }} M_{\Gamma, \Gamma^{\prime}}^{\langle n, m\rangle}-\sum_{\Gamma^{\prime} k \text {-odd }} M_{\Gamma, \Gamma^{\prime}}^{\langle n, m\rangle}$, or equivalently

$$
b_{\Gamma}=\#\left\{\Gamma^{\prime} k \text {-even } \mid \delta\left(\Gamma, \Gamma^{\prime}\right)=n-1\right\}-\#\left\{\Gamma^{\prime} k \text {-odd } \mid \delta\left(\Gamma, \Gamma^{\prime}\right)=n-1\right\} .
$$

If either of these sets is nonempty, then either one of the pairs $\{1,2\}, \ldots,\{2 k-1,2 k\}$ has both elements in $\Gamma$, or one of the pairs does not intersect $\Gamma$ and $\Gamma$ contains at least $n-k+1$ elements of $\{2 k+1, \ldots, n+m-1\}$. In the first case, we may construct $\Gamma^{\prime}$ by replacing either element of the pair; in the second case, we construct $\Gamma^{\prime}$ by replacing any of the extra elements from $\{2 k+1, \ldots, n+m-1\}$ with either element of the pair which does not intersect $\Gamma$. In both cases the choice of an element from the pair in question determines the $k$-parity of $\Gamma^{\prime}$, and since we may choose either one freely it is clear that there are equal numbers of $k$-even and $k$-odd $\Gamma^{\prime}$. In particular, this means that $b_{\Gamma}=0$ whenever $a_{k, \Gamma}=0$.

If instead $\Gamma$ is $k$-even, then $b_{\Gamma}=n+\#\left\{\Gamma^{\prime} k\right.$-even $\left.\mid \delta\left(\Gamma, \Gamma^{\prime}\right)=n-1\right\}-\#\left\{\Gamma^{\prime} k\right.$-odd $\mid$ $\left.\delta\left(\Gamma, \Gamma^{\prime}\right)=n-1\right\}$. The only way to construct $k$-even $\Gamma^{\prime}$ with $\delta\left(\Gamma, \Gamma^{\prime}\right)=n-1$ is to replace one of the $n-k$ elements in $\{2 k+1, \ldots, n+m-1\} \cap \Gamma$ with one of the $m-k-1$ elements in $\{2 k+1, \ldots, n+m-1\} \backslash \Gamma$, so there are $(n-k)(m-k-1)$ such $\Gamma^{\prime}$. In order to construct $k$-odd $\Gamma^{\prime}$ with $\delta\left(\Gamma, \Gamma^{\prime}\right)=n-1$, we must choose one of the $k$ pairs $\{1,2\}, \ldots,\{2 k-1,2 k\}$ and replace the element of that pair in $\Gamma$ with the other element, hence clearly there are $k$ such $\Gamma^{\prime}$. Therefore

$$
b_{\Gamma}=n+(n-k)(m-k-1)-k=(n-k)(m-k) .
$$

An identical computation yields $b_{\Gamma}=-(n-k)(m-k)$ when $\Gamma$ is $k$-odd, so $M^{\langle n, m\rangle} v_{k}=$ $(n-k)(m-k) \cdot v_{k}$ as desired.

Theorem 2.3. The matrix $M^{\langle n, m\rangle}$ is invertible for all $n \geq m \geq 2$. Therefore $K^{\langle n, m\rangle}$ has full rank.

Proof. None of the eigenvalues of $M^{\langle n, m\rangle}$ are zero.

Although we have computed all of the eigenvalues of $M^{\langle n, m\rangle}$, we have not actually shown how to diagonalize it because we do not know the multiplicities of each eigenvalue. The same series of computations which initially suggested Proposition 2.2 seems to support the following conjecture: 
Conjecture 2.4. The $\mathcal{S}_{n+m-1}$-orbit of $v_{k}$ spans a subspace of $H^{\langle n, m\rangle}$ of dimension equal to $\left(\begin{array}{c}n+m-1 \\ k\end{array}\right)-\left(\begin{array}{c}n+m-1 \\ k-1\end{array}\right)$. These subspaces are precisely the eigenspaces with eigenvalues $(n-k)(m-k)$.

Finally, we note that the matrices $K^{\langle n, n\rangle}$ are square and symmetric, so $M^{\langle n, n\rangle}=$ $\left(K^{\langle n, n\rangle}\right)^{2}$. In this case the eigenvalues of $M^{\langle n, n\rangle}$ all have the form $(n-k)^{2}$, so in fact $K^{\langle n, n\rangle}$ has integer eigenvalues. Also, if Conjecture 2.4 is true, then $M^{\langle n, n\rangle}$ has 1 as an eigenvalue with multiplicity $\left(\begin{array}{c}2 n-1 \\ n-1\end{array}\right)-\left(\begin{array}{c}2 n-1 \\ n-2\end{array}\right)=\frac{1}{n+1}\left(\begin{array}{c}2 n \\ n\end{array}\right)$, which is the $n$th Catalan number.

\section{Young diagrams with two rows}

In this section we will consider all Young diagrams of the shape $\lambda=(n, m)$, where $n>m \geq 1$. This does not cover precisely all shapes with two rows, since it omits $2 \times n$ rectangles $\lambda=(n, n)$, but this case was already solved by diagonalizing $M^{\lambda}$ in [2] and by a short combinatorial argument in [5].

Proposition 3.1. The matrices $K^{(n, n)}$ and $K^{(n, n-1)}$ are identical for all $n \geq 2$.

Proof. We construct bijections $f: H^{(n, n)} \rightarrow H^{(n, n-1)}$ and $g: V^{(n, n)} \rightarrow V^{(n, n-1)}$ by taking tabloids $\Gamma \in H^{(n, n)}$ or $\Delta \in V^{(n, n)}$, removing the square containing $2 n$ from its respective row or column, and possibly reordering the rows or columns of the resulting shape so that the shortened one appears last. It is clear that $\Gamma \perp \Delta$ if and only if $f(\Gamma) \perp g(\Delta)$, so $K_{\Gamma, \Delta}^{(n, n)}=K_{f(\Gamma), g(\Delta)}^{(n, n-1)}$ for all $\Gamma$ and $\Delta$.

Since $K^{(n, n)}$ has full rank, it follows that $K^{(n, n-1)}$ has full rank as well and thus we may now restrict our analysis to the case $m \leq n-2$.

Given $n>m$, we can define a function $\delta$ on pairs of row tabloids of shape $(n, m)$ just as in the case of hooks, by letting $\delta\left(\Gamma_{1}, \Gamma_{2}\right)$ be the number of elements which appear in the top row of both tabloids; note that we have $n-m \leq \delta\left(\Gamma_{1}, \Gamma_{2}\right) \leq n$. Suppose that $\delta\left(\Gamma_{1}, \Gamma_{2}\right)=k$, and without loss of generality assume that the top rows of $\Gamma_{1}$ and $\Gamma_{2}$ are $\{1,2, \ldots, n\}$ and $\{1, \ldots, k, n+1, \ldots, 2 n-k\}$ respectively. In a column tabloid $\Delta \in V^{(n, m)}$ which is orthogonal to both $\Gamma_{1}$ and $\Gamma_{2}$, any element in one of the 1-element columns must be in the top row of each $\Gamma_{i}$, or else two elements from the top row of one $\Gamma_{i}$ will end up in the same column somewhere else; there are thus $\left(\begin{array}{c}k \\ n-m\end{array}\right)$ ways to fill these columns. The remaining $k-n+m$ elements of $\{1,2, \ldots, k\}$ must be paired with elements of $\{2 n-k+1, \ldots, n+m\}$ in 2-element columns of $\Delta$, and there are $(k-n+m)$ ! ways to do so. Finally, the elements $\{k+1, \ldots, n\}$ from the top row of $\Gamma_{1}$ must be paired with $\{n+1, \ldots, 2 n-k\}$ in the remaining 2-element columns of $\Delta$, and this can be done in $(n-k)$ ! ways. Therefore there are a total of $\left(\begin{array}{c}k \\ n-m\end{array}\right) \cdot(k-n+m) ! \cdot(n-k)$ ! column tabloids orthogonal to both $\Gamma_{1}$ and $\Gamma_{2}$, or

$$
M_{\Gamma_{1}, \Gamma_{2}}^{(n, m)}=\frac{k !(n-k) !}{(n-m) !}=\frac{n !}{(n-m) !}\left(\begin{array}{l}
n \\
k
\end{array}\right)^{-1}
$$

whenever $\delta\left(\Gamma_{1}, \Gamma_{2}\right)=k$. 
Lemma 3.2. The minimal polynomial of $M^{(n, m)}$ has degree at most $m+1$ for all $n>$ $m \geq 1$.

Proof. We proceed exactly as in the proof of Lemma 2.1 for the case $\langle n, m+1\rangle$, noting that $\delta\left(\Gamma_{1}, \Gamma_{2}\right)$ can take any of the $m+1$ values $n, n-1, \ldots, n-m$.

Proposition 3.3. The eigenvectors $v_{k}, 0 \leq k \leq m$, of $M^{\langle n, m+1\rangle}$ which were constructed in Proposition 2.2 are also eigenvectors of $M^{(n, m)}$ for $n>m$.

Proof. We recall the construction of $v_{k}=\left(a_{k, \Gamma}\right)$, again referring to row tabloids $\Gamma \in H^{(n, m)}$ by the elements in their top rows (hence as $n$-element subsets of $\{1,2, \ldots, n+m\}$ ): For $0 \leq k \leq m$ we declare an $n$-element subset of $\{1, \ldots, n+m\}$ to be $k$-ordinary if it contains either zero or two elements from any of the pairs $\{1,2\},\{3,4\}, \ldots,\{2 k-1,2 k\}, k$-even if there are an even number of odd representatives from these pairs, and $k$-odd if the number of odd representatives is odd. We set $a_{k, \Gamma}=0$ if $\Gamma$ is $k$-ordinary; otherwise we set $a_{k, \Gamma}=1$ if $\Gamma$ is $k$-even and $a_{k, \Gamma}=-1$ if it is $k$-odd.

The proof that each $v_{k}$ is an eigenvector proceeds mostly as before, but with a little more attention to detail. Indeed, if $\Gamma$ is $k$-ordinary then it contains either both elements of a pair $\{2 i-1,2 i\}$ or neither of them, and then for fixed $d$ there is a bijection between the non- $k$-ordinary tabloids $\Gamma^{\prime}$ with $\delta\left(\Gamma, \Gamma^{\prime}\right)=d$ which are $k$-even and those which are $k$-odd: half contain $2 i-1$ and the other half contain $2 i$. Since these $k$-even and $k$-odd tabloids contribute equally to the sum $\sum_{\Gamma^{\prime}} M_{\Gamma, \Gamma^{\prime}}^{(n, m)} a_{k, \Gamma^{\prime}}$ but with opposite signs, the sum is zero.

If $\Gamma$ is $k$-even, then we can compute

$$
\sum_{\Gamma^{\prime}} M_{\Gamma, \Gamma^{\prime}}^{(n, m)} a_{k, \Gamma^{\prime}}=\sum_{d=0}^{m} \frac{d !(n-d) !}{(n-m) !} \sum_{\delta\left(\Gamma, \Gamma^{\prime}\right)=n-d} a_{k, \Gamma^{\prime}}
$$

For fixed $d$, the sum $\sum a_{k, \Gamma^{\prime}}$ is simply the difference between the number of $k$-even $\Gamma^{\prime}$ and the number of $k$-odd $\Gamma^{\prime}$. Any $\Gamma^{\prime}$ with $\delta\left(\Gamma, \Gamma^{\prime}\right)=n-d$ which is not $k$-ordinary is constructed by fixing $i \leq d$, picking $i$ of the $k$ pairs $\{1,2\}, \ldots,\{2 k-1,2 k\}$ and replacing the element of $\Gamma$ in each chosen pair with the other element of that pair, and then replacing $d-i$ of the $n-k$ elements of $\{2 k+1, \ldots, n+m\} \cap \Gamma$ with $d-i$ of the $m-k$ elements of $\{2 k+1, \ldots, n+m\} \backslash \Gamma$. For fixed $i$ this can be done in $\left(\begin{array}{c}k \\ i\end{array}\right)\left(\begin{array}{c}n-k \\ d-i\end{array}\right)\left(\begin{array}{c}m-k \\ d-i\end{array}\right)$ ways, and the resulting $\Gamma^{\prime}$ is $k$-even if $i$ is even and $k$-odd otherwise, so we get

$$
\sum_{\Gamma^{\prime}} M_{\Gamma, \Gamma^{\prime}}^{(n, m)} a_{k, \Gamma^{\prime}}=\sum_{d=0}^{m} \frac{d !(n-d) !}{(n-m) !} \sum_{i=0}^{d}(-1)^{i}\left(\begin{array}{c}
k \\
i
\end{array}\right)\left(\begin{array}{c}
n-k \\
d-i
\end{array}\right)\left(\begin{array}{c}
m-k \\
d-i
\end{array}\right) .
$$

The sum on the right side is independent of the $k$-even tabloid $\Gamma$, and we get an identical result for $k$-odd $\Gamma$, so $v_{k}$ is indeed an eigenvector of $M^{(n, m)}$ with eigenvalue $\lambda_{k}$ equal to the sum (1).

We now attempt to rearrange equation (1) somewhat in order to compare the values of $\lambda_{k}$; our goal will be to prove that $\lambda_{0}>\lambda_{1}>\cdots>\lambda_{m}>0$. Letting $j=d-i$ and 
expanding the binomial coefficients in order to rearrange them, we get

$$
\begin{aligned}
\lambda_{k} & =\sum_{d=0}^{m} \frac{d !(n-d) !}{(n-m) !} \sum_{j=0}^{d}(-1)^{d-j} \frac{k !}{(d-j) !(k-d+j) !} \cdot \frac{(n-k) !}{j !(n-k-j) !} \cdot\left(\begin{array}{c}
m-k \\
j
\end{array}\right) \\
& =\sum_{d=0}^{m} \frac{k !(n-k) !}{(n-m) !} \sum_{j=0}^{d}(-1)^{d+j} \frac{d !}{j !(d-j) !} \cdot \frac{(n-d) !}{(n-k-j) !(k-d+j) !} \cdot\left(\begin{array}{c}
m-k \\
j
\end{array}\right) \\
& =\frac{k !(n-k) !}{(n-m) !} \sum_{d=0}^{m} \sum_{j=0}^{d}(-1)^{d+j}\left(\begin{array}{l}
d \\
j
\end{array}\right)\left(\begin{array}{c}
n-d \\
n-k-j
\end{array}\right)\left(\begin{array}{c}
m-k \\
j
\end{array}\right) .
\end{aligned}
$$

We can replace the upper limit $j \leq d$ with $j \leq \infty$ without changing $\lambda_{k}$, since any term with $j>d$ will have $\left(\begin{array}{l}d \\ j\end{array}\right)=0$. Likewise we can replace $d \leq m$ with $d \leq n$ : If $m<d \leq n$ and $\left(\begin{array}{c}n-d \\ n-k-j\end{array}\right) \neq 0$ then $n-k-j \leq n-d$, hence $m-k \leq m-d+j<j$ and so $\left(\begin{array}{c}m-k \\ j\end{array}\right)=0$. Therefore we define

$$
f(m, n, k)=\sum_{d=0}^{n} \sum_{j=0}^{\infty}(-1)^{d+j}\left(\begin{array}{l}
d \\
j
\end{array}\right)\left(\begin{array}{c}
n-d \\
n-k-j
\end{array}\right)\left(\begin{array}{c}
m-k \\
j
\end{array}\right)
$$

and note that $\lambda_{k}=\frac{k !(n-k) !}{(n-m) !} f(m, n, k)$.

We will now investigate the values of $f(m, n, k)$ by making extensive use of generating functions. Let $a=m-k, b=n-k$, and $c=n$, and define $F(x, y, z)=$ $\sum_{m, n} \sum_{k=0}^{\min (m, n)} f(m, n, k) x^{m-k} y^{n-k} z^{n} ;$ then

$$
\begin{aligned}
F(x, y, z) & =\sum_{\substack{a, b, c \\
b \leq c \\
b=0}}^{c} \sum_{j=0}^{\infty}(-1)^{d+j}\left(\begin{array}{l}
d \\
j
\end{array}\right)\left(\begin{array}{l}
c-d \\
b-j
\end{array}\right)\left(\begin{array}{l}
a \\
j
\end{array}\right) x^{a} y^{b} z^{c} \\
& =\sum_{\substack{a, b, c=0 \\
\infty}}^{c} \sum_{d=0}^{\infty}(-1)^{d+j}\left(\begin{array}{l}
d \\
j
\end{array}\right)\left(\begin{array}{l}
c-d \\
b-j
\end{array}\right)\left(\begin{array}{l}
a \\
j
\end{array}\right) x^{a} y^{b} z^{c} \\
& =\sum_{d=0}^{\infty} \sum_{j=0}^{\infty} \sum_{\substack{a, b, c \\
c \geq d}}(-1)^{d+j}\left(\begin{array}{l}
d \\
j
\end{array}\right)\left(\begin{array}{l}
c-d \\
b-j
\end{array}\right)\left(\begin{array}{l}
a \\
j
\end{array}\right) x^{a} y^{b} z^{c}
\end{aligned}
$$

where we can remove the condition $b \leq c$ as follows: Consider a potential term in this sum for which $b>c$. If this term is nonzero then $\left(\begin{array}{l}d \\ j\end{array}\right) \neq 0$ implies $j \leq d$, but then $c-d<b-d \leq b-j$ and so $\left(\begin{array}{c}c-d \\ b-j\end{array}\right)$ is zero anyway. Having exchanged the order of 
summation, we simplify:

$$
\begin{aligned}
F(x, y, z) & =\sum_{d=0}^{\infty} \sum_{j=0}^{\infty} \sum_{\substack{b, c \\
c \geq d}}(-1)^{d+j}\left(\begin{array}{l}
d \\
j
\end{array}\right) \frac{x^{j}}{(1-x)^{j+1}} y^{j} \cdot\left(\begin{array}{l}
c-d \\
b-j
\end{array}\right) y^{b-j} z^{c} \\
& =\sum_{d=0}^{\infty} \sum_{j=0}^{\infty} \sum_{c=d}^{\infty}(-1)^{d+j}\left(\begin{array}{l}
d \\
j
\end{array}\right) \frac{x^{j} y^{j}}{(1-x)^{j+1}} z^{d} \cdot(1+y)^{c-d} z^{c-d} \\
& =\sum_{d=0}^{\infty} \sum_{j=0}^{\infty} \frac{(-1)^{d}}{1-x} \cdot\left(\begin{array}{l}
d \\
j
\end{array}\right)\left(\frac{-x y}{1-x}\right)^{j} \frac{z^{d}}{1-z(1+y)} \\
& =\sum_{d=0}^{\infty}(-1)^{d}\left(1-\frac{x y}{1-x}\right)^{d} \frac{z^{d}}{(1-x)(1-z(1+y))} \\
& =\frac{1}{(1-x)(1-z(1+y))} \cdot \frac{1}{1+z\left(1-\frac{x y}{1-x}\right)} \\
& =\frac{1}{1-z-y z} \cdot \frac{1}{1-x+z-x z-x y z}
\end{aligned}
$$

Here we have eliminated the indices of summation $a, b, c, j$, and $d$ in that order by using the identity

$$
\sum_{a=0}^{\infty}\left(\begin{array}{l}
a \\
j
\end{array}\right) x^{a}=\frac{x^{j}}{(1-x)^{j+1}}
$$

in addition to the binomial theorem and repeated summation of geometric series.

Making the substitution $w=y z$, we now have

$$
\begin{aligned}
F(x, y, z) & =\frac{1}{1-x+z-x z-x w} \cdot \frac{1}{1-z-w} \\
& =\frac{1 /(1+z)}{1-x\left(1+\frac{w}{1+z}\right)} \cdot \frac{1}{1-z-w} \\
& =\sum_{a=0}^{\infty} x^{a} \cdot \frac{1}{(1+z)(1-z-w)}\left(1+\frac{w}{1+z}\right)^{a} \\
& =\sum_{a=0}^{\infty} x^{a}\left(\sum_{p=0}^{\infty} \frac{w^{p}}{(1-z)^{p+1}}\right)\left(\sum_{q=0}^{a}\left(\begin{array}{l}
a \\
q
\end{array}\right) \frac{w^{q}}{(1+z)^{q+1}}\right)
\end{aligned}
$$

and so the coefficient of $x^{a} w^{b}=x^{m-k} w^{n-k}$ in $F$ is

$$
\begin{aligned}
C_{a b}(z) & =\frac{1}{1-z^{2}} \cdot \sum_{p+q=n-k}\left(\begin{array}{c}
m-k \\
q
\end{array}\right)\left(\frac{1}{1+z}\right)^{q}\left(\frac{1}{1-z}\right)^{p} \\
& =\frac{1}{1-z^{2}} \cdot \sum_{q=0}^{n-k}\left(\begin{array}{c}
m-k \\
q
\end{array}\right)\left(\frac{1}{1+z}\right)^{q}\left(\frac{1}{1-z}\right)^{n-k-q} .
\end{aligned}
$$


Now we should note that the only values of $f$ we really wish to compute are those for which $n>m$, since we are analyzing the eigenvalues $\lambda_{k}$ for $n>m$. Thus we choose to modify this sum by replacing the upper limit $q=n-k$ with $q=m-k$, since when $m \leq n$ it makes no difference. Calling the modified sum $\tilde{C}_{a b}(z)$, we have

$$
\begin{aligned}
\tilde{C}_{a b}(z) & =\frac{1}{1-z^{2}}\left(\frac{1}{1-z}\right)^{n-k} \cdot \sum_{q=0}^{m-k}\left(\begin{array}{c}
m-k \\
q
\end{array}\right)\left(\frac{1-z}{1+z}\right)^{q} \\
& =\frac{1}{1-z^{2}}\left(\frac{1}{1-z}\right)^{n-k}\left(1+\frac{1-z}{1+z}\right)^{m-k} \\
& =\frac{2^{m-k}}{1-z^{2}}\left(\frac{1}{1-z}\right)^{n-k}\left(\frac{1}{1+z}\right)^{m-k} .
\end{aligned}
$$

At this point we should pause for a moment to summarize the current situation. The function $\tilde{F}(x, y, z)=\sum_{a, b} \tilde{C}_{a b}(z) \cdot x^{a} w^{b}$, where $w=y z$, is not a generating function for $f(m, n, k)$ but for some other $\tilde{f}(m, n, k)$. However, when $m \leq n$, the coefficient of $x^{m-k} y^{n-k} z^{n}=x^{m-k} w^{n-k} z^{k}$ is still $f(m, n, k)$, and thus we may proceed with our analysis. In fact, extracting the $z^{k}$ coefficient from $\tilde{C}_{a b}(z)$ allows us to prove the following.

Lemma 3.4. If $n>m$ then $\lambda_{k}$ is positive for all $k, 0 \leq k \leq m$.

Proof. Recall that $\lambda_{k}=\frac{k !(n-k) !}{(n-m) !} f(m, n, k)$. We rewrite $\tilde{C}_{a b}(z)$ slightly in order to find its $z^{k}$-coefficient:

$$
\begin{aligned}
\tilde{C}_{a b}(z) & =2^{m-k}\left(\frac{1}{1-z^{2}}\right)^{m-k+1}\left(\frac{1}{1-z}\right)^{n-m} \\
& =2^{m-k}\left(\sum_{i=0}^{\infty}\left(\begin{array}{c}
-(m-k+1) \\
i
\end{array}\right)\left(-z^{2}\right)^{i}\right)\left(\sum_{j=0}^{\infty}\left(\begin{array}{c}
-(n-m) \\
j
\end{array}\right)(-z)^{j}\right)
\end{aligned}
$$

and since $\left(\begin{array}{c}-p \\ q\end{array}\right)=(-1)^{q}\left(\begin{array}{c}p+q-1 \\ q\end{array}\right)$, the desired coefficient is

$$
\begin{aligned}
f(m, n, k) & =2^{m-k} \sum_{2 i+j=k}\left(\begin{array}{c}
m-k+i \\
i
\end{array}\right)\left(\begin{array}{c}
n-m+j-1 \\
j
\end{array}\right) \\
& =2^{m-k} \sum_{i=0}^{\lfloor k / 2\rfloor}\left(\begin{array}{c}
m-k+i \\
i
\end{array}\right)\left(\begin{array}{c}
n-m+k-2 i-1 \\
k-2 i
\end{array}\right) .
\end{aligned}
$$

Every term here is nonnegative, and the $i=0$ term is $\left(\begin{array}{c}m-k \\ 0\end{array}\right)\left(\begin{array}{c}n-m+k-1 \\ k\end{array}\right)=\left(\begin{array}{c}n-(m+1)+k \\ k\end{array}\right)$, which is positive as long as $n \geq m+1$, so $f(m, n, k)$ and hence $\lambda_{k}$ are positive.

Remark 3.5. Equation (2) certainly gives a nicer form for $\lambda_{k}$ than the one in equation (1), since there is only one index of summation and all terms are nonnegative. For example, we may use it to immediately compute $\lambda_{0}=\frac{2^{m} \cdot n !}{(n-m) !}$ and $\lambda_{1}=\frac{2^{m-1} \cdot(n-1) !}{(n-m-1) !}$, and in the case $\lambda=(n, 1)=\langle n, 2\rangle$ we see once again that the eigenvalues are $2 n$ and $n-1$. 
When $n=m+1$ we can even find a closed form for all of the $\lambda_{k}$ :

$$
\begin{aligned}
f(n-1, n, k) & =2^{(n-1)-k} \sum_{i=0}^{\lfloor k / 2\rfloor}\left(\begin{array}{c}
(n-1)-k+i \\
i
\end{array}\right)\left(\begin{array}{c}
n-(n-1)+k-2 i-1 \\
k-2 i
\end{array}\right) \\
& =2^{n-1-k} \sum_{i=0}^{\lfloor k / 2\rfloor}\left(\begin{array}{c}
(n-1-k)+i \\
i
\end{array}\right)
\end{aligned}
$$

and the identity $\sum_{i=0}^{p}\left(\begin{array}{c}q+i \\ i\end{array}\right)=\left(\begin{array}{c}p+q+1 \\ p\end{array}\right)$ lets us simplify the sum to $\left(\begin{array}{c}n-k+\lfloor k / 2\rfloor \\ \lfloor k / 2\rfloor\end{array}\right)=\left(\begin{array}{c}n-\lceil k / 2\rceil \\ \lfloor k / 2\rfloor\end{array}\right)$, SO

$$
\lambda_{k}=k !(n-k) ! \cdot 2^{n-1-k}\left(\begin{array}{c}
n-\lceil k / 2\rceil \\
\lfloor k / 2\rfloor
\end{array}\right)
$$

for $n=m+1$. This matches the computed eigenvalues for $M^{(n, n)}$ in [2], which is no surprise since $K^{(n, n-1)}$ and $K^{(n, n)}$ are identical; in particular, one can check that $\lambda_{0}>$ $\lambda_{1}=\lambda_{2}>\lambda_{3}=\lambda_{4}>\ldots$, but our list of eigenvalues is complete even though we only have $\left\lceil\frac{m}{2}\right\rceil+1$ distinct values. It is also shown in [2] that the eigenspaces for $\lambda_{2 i-1}=\lambda_{2 i}$ have dimension $\left(\begin{array}{c}2 n-1 \\ 2 i\end{array}\right)-\left(\begin{array}{c}2 n-1 \\ 2 i-2\end{array}\right)=\left[\left(\begin{array}{c}2 n-1 \\ 2 i-1\end{array}\right)-\left(\begin{array}{c}2 n-1 \\ 2 i-2\end{array}\right)\right]+\left[\left(\begin{array}{c}2 n-1 \\ 2 i\end{array}\right)-\left(\begin{array}{c}2 n-1 \\ 2 i-1\end{array}\right)\right]$, which supports Conjecture 2.4.

Define a new function $g(m, n, k)=\frac{\tilde{f}(m, n, k)}{2^{m-k}}$, noting that we are specifically using the modified $\tilde{f}$, and let

$$
\begin{aligned}
G(x, w, z) & =\sum_{m, n \geq k} g(m, n, k) \cdot x^{m-k} w^{n-k} z^{k} \\
& =\sum_{a, b} \frac{\tilde{C}_{a b}(z)}{2^{m-k}} x^{a} w^{b} \\
& =\sum_{a, b} \frac{1}{1-z^{2}}\left(\frac{1}{1-z}\right)^{b}\left(\frac{1}{1+z}\right)^{a} x^{a} w^{b} \\
& =\frac{1}{1-z^{2}} \frac{1}{1-\frac{x}{1+z}} \frac{1}{1-\frac{w}{1-z}} \\
& =\frac{1}{(1+z-x)(1-z-w)} .
\end{aligned}
$$

Now $\lambda_{k}>\lambda_{k+1}$ if and only if $\frac{k !(n-k) !}{(n-m) !} f(m, n, k)>\frac{(k+1) !(n-k-1) !}{(n-m) !} f(m, n, k+1)$, and since $f(m, n, k)=2^{m-k} g(m, n, k)$ this is equivalent to

$$
2(n-k) g(m, n, k)>(k+1) g(m, n, k+1) .
$$

Noting that $2(n-k) g(m, n, k)$ is the $x^{m-k} w^{n-k} z^{k}$-coefficient of $2 w \frac{\partial G}{\partial w}$ and $(k+1) g(m, n, k+$ 1 ) is the $x^{m-k} w^{n-k} z^{k}$-coefficient of $x w \frac{\partial G}{\partial z}$, we conclude that $\lambda_{k}>\lambda_{k+1}$ iff the function $H(x, w, z)=2 \frac{\partial G}{\partial w}-x \frac{\partial G}{\partial z}$ has a positive $x^{m-k} w^{n-k-1} z^{k}$-coefficient. 
We begin to evaluate $H$ :

$$
\begin{aligned}
H(x, w, z) & =\frac{2(1+z-x)-x(1+z-x)+x(1-z-w)}{(1+z-x)^{2}(1-z-w)^{2}} \\
& =\left(2+2 z-2 x-2 x z+x^{2}-x w\right)\left(\frac{1 /(1+z)}{1-\frac{x}{1+z}}\right)^{2}\left(\frac{1 /(1-z)}{1-\frac{w}{1-z}}\right)^{2} \\
& =\frac{2(1+z)-2 x(1+z)+x^{2}-x w}{\left(1-z^{2}\right)^{2}} \cdot \sum_{p, q \geq 1} p q\left(\frac{x}{1+z}\right)^{p-1}\left(\frac{w}{1-z}\right)^{q-1}
\end{aligned}
$$

where we use the identity $\left(\frac{1}{1-r}\right)^{2}=\sum_{i \geq 1} i r^{i-1}$. The coefficient of $x^{\alpha-1} w^{\beta-1}$ in the function $\left(1-z^{2}\right)^{2}(1+z)^{\alpha-1}(1-z)^{\beta-1} H(x, w, z)$ is therefore

$2(1+z) \alpha \beta-2(1+z)(\alpha-1) \beta(1+z)+(1+z)^{2}(\alpha-2) \beta-(\alpha-1)(\beta-1)(1+z)(1-z)$

when $\alpha \geq 2$ and $\beta \geq 1$. This simplifies to $(\alpha+\beta-1)\left(1-z^{2}\right)$, so equivalently $H(x, w, z)$ has $x^{\alpha-1} w^{\beta-1}$-coefficient (as a function of $z$ ) equal to

$$
\frac{\alpha+\beta-1}{(1+z)^{\alpha}(1-z)^{\beta}} \text {. }
$$

Now if $\beta \geq \alpha$ then the denominator can be written $\left(1-z^{2}\right)^{\alpha}(1-z)^{\beta-\alpha}$, and so this coefficient equals

$$
(\alpha+\beta-1)\left(1+z^{2}+z^{4}+\ldots\right)^{\alpha}\left(1+z+z^{2}+\ldots\right)^{\beta-\alpha} .
$$

Clearly this power series in $z$ has positive $z^{k}$-coefficients for all $k \geq 0$ whenever $\beta>\alpha$, and when $\beta=\alpha$ these coefficients alternate between positive values and zero. Letting $\alpha=m-k+1$ and $\beta=n-k$, and noting that $k<m<n-1$ implies $\beta>\alpha \geq 2$, we can conclude:

Proposition 3.6. If $n>m+1$ then $\lambda_{0}>\lambda_{1}>\cdots>\lambda_{m}$.

The fact that the coefficients alternate between positive values and zero when $\beta=\alpha$ proves again that $\lambda_{0}>\lambda_{1}=\lambda_{2}>\lambda_{3}=\lambda_{4}>\ldots$ when $n=m+1$.

Theorem 3.7. The matrices $M^{(n, m)}$ are invertible for $n-2 \geq m \geq 1$.

Proof. Recall that by Lemma 3.2, the minimal polynomial of $M^{(n, m)}$ has degree at most $m+1$. The $m+1$ eigenvalues $\lambda_{k}, 0 \leq k \leq m$, are distinct by Proposition 3.6, so they are all of the eigenvalues of $M^{(n, m)}$. But the $\lambda_{k}$ are also positive by Lemma 3.4, and since $M^{(n, m)}$ has only positive eigenvalues it must be invertible.

The remaining cases $n=m$ and $n=m+1$ are already done, so this completes the analysis for all Young diagrams with two rows.

Corollary 3.8. The $\mathcal{S}_{|\lambda|}$-module homomorphism $\left(K^{\lambda}\right)^{\top}$ realizes $\mathbb{Q} H^{\lambda}$ as a submodule of $\mathbb{Q} H^{\lambda^{\prime}}$ for all diagrams $\lambda=(n, m)$. 


\section{Open questions}

For which Young diagrams $\lambda$ is $\mathbb{Q} H^{\lambda}$ a submodule of $\mathbb{Q} H^{\lambda^{\prime}}$ ? A complete answer even for $\lambda$ having just three rows will be hard to find by the method in this paper, not just because the matrices will be harder to analyze but because $K^{(4,3,3)}$ does not have full rank [6]. However, an analysis of $M^{\lambda}$ would still be interesting in this case, or even restricted to the cases $\lambda=(n, n, n)$ or $\lambda=(n, m, k)$ for $n \geq m+1 \geq k+2$. For example, some computations using Sage and LinBox have completely determined the eigenvalues of $M^{(4,4,4)}$ :

Fact 4.1. The characteristic polynomial of $M^{(4,4,4)}$ is

$$
\begin{aligned}
f(\lambda)= & (\lambda-124416)(\lambda-10368)^{54}(\lambda-3456)^{154}(\lambda-2304)^{275}(\lambda-768)^{132} \\
& \cdot(\lambda-576)^{1408}(\lambda-288)^{616}(\lambda-128)^{2673}(\lambda-96)^{462} .
\end{aligned}
$$

Note that these eigenvalues all have the form $2^{a} 3^{b}$; is there an explanation for this that might extend to other $n \times 3$ rectangles?

Coker [2] suggests an algorithm to diagonalize $M^{(n, n, n)}$ and carries it out for $n \leq 6$; we note that for $M^{(5,5,5)}$ the eigenvalues are not all integers. However, even when they are not integers it should not be hard, using arguments similar to those of Lemmas 2.1 and 3.2, to devise an algorithm which computes the minimal polynomial of $M^{\lambda}$ for more general $\lambda$. This algorithm should be simpler in practice because it does not need to determine multiplicity information to decide whether $M^{\lambda}$ is invertible. In some cases this may hopefully lead to a worthwhile analysis of $M^{\lambda}$ for a larger class of diagrams $\lambda$.

Acknowledgement. Both authors are supported by NDSEG graduate fellowships.

\section{References}

[1] S.C. Black and R.J. List, A note on plethysm, Euro. J. Combin. 10 (1989), 111-112.

[2] C. Coker, A problem related to Foulkes's conjecture, Graphs Combin. 9 (1993), 117134.

[3] J.-G. Dumas et al., LinBox, http://www.linalg.org/.

[4] J. Mller and M. Neunhffer, Some computations regarding Foulkes' conjecture, Experiment. Math. 14 (2005), no. 3, 277-283.

[5] P. Pylyavskyy, On plethysm conjectures of Stanley and Foulkes: the $2 \times n$ case, Electron. J. Combin. 11(2) (2004), \#R8.

[6] S. Sivek, Some plethysm results related to Foulkes' conjecture, Electron. J. Combin. 13 (2006), \#R24.

[7] R. Stanley, Positivity problems and conjectures in algebraic combinatorics, Mathematics: Frontiers and Perspectives, Amer. Math. Soc., Providence, RI, 2000, pp. 295-319.

[8] W. Stein, Sage Mathematics Software (Version 2.9.3), The Sage Group, 2008, http: //www . sagemath.org.

[9] H. Wilf, generatingfunctionology, 2nd edition, Academic Press, Boston, MA, 1994. 